\title{
Literature Survey on Recent Progress in Inter-Vehicle Communication Simulations
}

\author{
Markus Hager ${ }^{1}$, Jochen Seitz ${ }^{1}$, Thomas Waas ${ }^{2}$ \\ ${ }^{1}$ Fachgebiet Kommunikationsnetze, Technische Universität IImenau, Ilmenau, Germany \\ ${ }^{2}$ Labor Computernetzwerke, Ostbayrische Technische Hochschule Regensburg, Regensburg, Germany \\ Email: markus.hager@tu-ilmenau.de, jochen.seitz@tu-ilmenau.de, thomas.waas@hs-regensburg.de
}

Received 13 April 2015; accepted 6 July 2015; published 13 July 2015

Copyright (C) 2015 by authors and Scientific Research Publishing Inc.

This work is licensed under the Creative Commons Attribution International License (CC BY).

http://creativecommons.org/licenses/by/4.0/

(c) (i) Open Access

\section{Abstract}

The vehicular ad hoc network (VANET) technology based on the approved IEEE 802.11p standard and the appendant inter-vehicle communication (IVC) has the potential to dramatically change the way transportation systems work. The fundamental idea is to change the individual behavior of each vehicle by exchanging information among traffic participants to realize a cooperative and more efficient transportation system. Certainly, the evaluation of such systems is a comprehensive and challenging task in a real world test bed, therefore, simulation frameworks are a key tool to analyze IVC. Several models are needed to emulate the real behavior of a VANET in all aspects as much realistically as necessary. The intention of this survey is to provide a comprehensive overview of publications concerning IVC simulations of the year 2013 and to see how IVC simulation has changed since 2009. Based on this analysis, we will answer the following questions: What simulation techniques are applied to IVC? Which aspects of IVS have been evaluated? What has changed within five years of IVC simulations? We also take a closer look at commonly used software tools and discuss their functionality and drawbacks. Finally, we present open questions concerning IVC simulations.

\section{Keywords}

Inter-Vehicle Communication, Simulation, Survey

\section{Introduction}

Looking at the development and utilization of information technology in vehicles, we can see an enormous progress and development during the last decades. Current vehicles have a large amount of sensors (e.g. radar, ultrasonic and cameras) to scan adjacency regions as well as rain and temperature sensors, too. Based on this 
data, an autonomously driving vehicle is already possible for most traffic situations. But due to legal constraints and open questions regarding the liability in the event of a failure, this technology is mostly used to support the driver or to react in critical situations as in case of an imminent rear-end collision. The combination of this sensor information and other context data with IVC leads to intelligent transportation systems (ITS). Kashif Dar et al. [1] described the structure and general applications of ITS and provided a comparison to other wireless communication systems, whereas Willke et al. [2] presented a more comprehensive discussion of this topic as well as d'Orey and Ferreira [3].

IVC is based on the IEEE standard 802.11p [4] which is a modified version of IEEE 802.11a. As in classical mobile communications, the situation is different in the US [5] and Europe [6], but in general, the principles are the same. The applications can be classified into safety, traffic efficiency and entertainment solutions. Apart from the latter, not a core element of ITS applications, the corresponding protocol mechanisms are based on beacons for vehicular safety and on multi-hop routing and forwarding schemes in case of traffic information. At this point, we want to exclude advanced techniques like further mobile communication systems or background Internet cloud services to the benefit of a clear focus on the VANET communication.

Beacons [7] [8] are broadcast messages periodically sent by each vehicle to inform about its position, speed, etc. and are not forwarded by other nodes of the VANET. Typical applications based on this scheme are emergency vehicle warnings or collision risk alerts. In case of an icy road segment or for traffic jam announcements, it is purposive to disseminate this information in a larger area to give road users the chance to take a different route in advance. Therefore, multi-hop broadcast protocols [9] are the second major communication component of ITS. The challenge is to forward these messages in such a way that all vehicles are aware of this event but keeping the amount of sending procedures as low as possible to save radio channel resources. Thus, a too selective strategy is faced with the risk that information gets completely lost whereas otherwise too many redundant messages are generated. For beacon based communications, even though no forwarding decision must be made, difficulties arise in case of high traffic density due to the cyclic unconditional sending process. Therefore, besides the recommendation of the standardization institutes, many modifications and extensions have been discussed in the literature [10] for these protocol schemes.

Field operational tests are one option to evaluate performance and reliability of IVC under real conditions. Examples are the finished $\operatorname{sim}^{\mathrm{TD}}$ project in Germany [11] or the DRIVEC2X project [12]. Such test beds are good for a proof of concept to demonstrate feasibility of IVC. Nevertheless, considering the reproducibility of field tests, it is in most cases impossible to setup identical conditions. But this would be necessary to improve statistical significance by repeating the process several times or to verify the impact of different protocols in the same situation. It would be difficult to independently control and repeat these experiments, too. For this reason, simulation frameworks are an important alternative for the development and evaluation process of inter-vehicle communication protocols. It is a simple task in case of a simulation to repeat an experiment several times to evaluate the stability of the system under varying conditions or to test several protocols in the same situation to compare them. But regarding the reproducibility in view of independent control, as it should be done for all scientific work, the situation is problematic as well. This has been figured out by the literature survey of StefanJoerer et al. [13]. The reason is that a simulation is based on several models, e.g. in case of IVC, a channel model is needed to describe the radio wave propagation, a mobility model for the movements of vehicles and the protocol or application logic itself. There are various software tools available to support this evaluation process. SUMO (simulation of urban mobility) [14] is one commonly used framework for mobility models supporting a good degree of realism. The same is true in case of radio signal propagation models or medium access schemes like the IEEE 802.11p standard. These models are quite complex and offer a large degree of freedom, therefore, in order to allow other researches to reproduce simulation results, it is necessary to clearly specify which model is used and which parameters values are set to configure these models.

The main contribution of this survey is to update the study done by Stefan Joerer et al. [13] by looking at publications on the topic of IVC concerning the accuracy description of the used models. Based on this analysis we discuss how the situation has changed in the year 2013 compared to 2009 until 2011. Additionally, we describe the most frequently used models and corresponding software tools in more details. Finally, we conclude this overview by presenting still remaining open questions in the field of ITS/IVC.

\section{Investigated Publications}

Even though the ideas and principles of IVC and ITS are not new, there is still a lot of research dealing with 
these topics. Not surprisingly, there are more than one hundred search hits for the key words "inter vehicle communication" only for the year 2013 in the IEEE Xplore database and several more publications on these topics could be found by choosing a query with synonyms. Therefore, to answer the question which publication should be counted for such a survey, we followed the idea of Stefan Joerer to look on some well-known conferences. These are:

- ACM VANET 2013 (Workshop on Vehicular Internetworking) held in conjunction with ACM MobiSys 2013 in Taipei, Taiwan. The workshop has a special focus on VANET issues, but besides this, a quite wide range of possible topics.

- IEEE VNC 2013 (Vehicular Networking Conference), Boston, USA. One of the most interesting conferences for this survey due to the focus on VANETs and IVC.

- IEEE VTC 2013 (Vehicular Technology Conference) spring (Dresden, Germany) and autumn (Las-Vegas, USA). May be one of the best known conferences but usually with most topics on radio channel models, measurements and medium access schemes.

Because the original study was based on a period of three years, whereas we look at only one year, we additionally included the following conferences for our study as well:

- ITST 2013 (Intelligent Transportation Systems Telecommunications), Tampere, Finland. The scope of this conference is on ITS with an alternating special issue which was in the year 2013 "Internet of Things".

- IEEE ITSC 2013 (Intelligent Transportation Systems Conference), the Hague, the Netherlands. A large conference with nearly four hundred publications on ITS.

In total, there have been more than 1300 papers published on these conferences in 2013. Please note that we have decided to skip a detailed evaluation of publications of the year 2012 because we think the trends in IVC form past years to now could also be seen based on an update of the last year. Furthermore, we did not include popular magazines and journals like the IEEE Communications Magazine or the IEEE Intelligent Transportation Magazine. The focus of these magazines is much broader and the style of the published articles is recommended to be written as a tutorial, therefore, the amount of papers meeting our requirements was really low resulting in our choice to include the two mentioned conferences instead.

For this survey, we only selected those papers with a clear focus on IVC simulations. Typical examples are the evaluation of IEEE 802.11p medium access modifications, VANET routing protocols, dissemination of traffic information or beaconing strategies. In contrast we excluded analytical models, design concepts, measurement studies and evaluations of channel models. The essential requirement to pass our selection process was the evaluation of IVC with the help of simulation. We found 38 publications meeting these requirements. Figure 1 illustrates the number of selected papers we have reviewed for each of the mentioned conferences in addition to the base data presented in [13]. Please note that we decided not to take into account the short papers of VNC

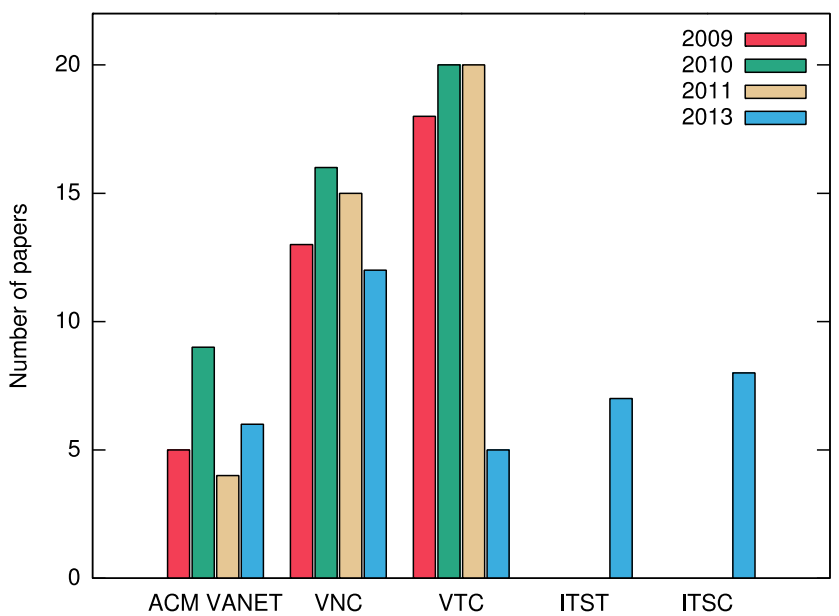

Figure 1. The number of reviewed papers meeting our requirements mentioned in section 2. Please note that zero in case of ITST and ITSC in years 2009 to 2011 means we did not investigate the amount of published papers for these years. 
2013. This is because of we think authors had to respect a page count limitation and therefore they may skipped some essential information they otherwise would have mentioned in their paper. The counting shows that only in case of the IEEE VTC conference, the amount of papers has decreased on these topics. This exception may be due to the typical focus on communication issues on lower levels like radio channel measurement studies, radio wave propagation models or coding schemes. Therefore, it could be said IVC is still in interest of research and it would be worth to look at the details how IVC has been evaluated via simulations to answer the questions if the situation has changed over the last years and what are the major tools to perform such an evaluation of IVC.

\section{Investigation Criteria and Rating}

Following the concept of [13], an IVC simulation is based on five elements:

1. Radio wave propagation: The physical model or more common channel model represents the characteristics of radio wave propagation. The most often used is a simple path loss model where the propagation loss depends only on the distance between transmitter and receiver. Other typical effects like shadowing and multi-path propagation are not part of this model.

2. Medium access: For IVC the IEEE 802.11p standard defines the medium access strategy and should therefore be used unless the medium access protocol itself and modifications of it are the object which is being evaluated in detail.

3. Mobility models: For mobile communication simulations, the node placement and movement is an important aspect because this mainly affects the interconnections between nodes, the complete network topology and therefore its fundamental characteristics.

4. Network simulator: In the field of communication engineering, there are some well-known and established network simulators. In case of IVC, these simulators would be the central tool which mainly influences which other existing software components and models could be used. Moreover, these software frameworks usually offer a large set of predefined elements to build up a simulation scenario implying a large set of parameters.

5. Scenario description: Because IVC is a wide field with lots of possible applications and scenarios each work should have a clearly defined scope.

To provide simulation results which could be reproduced by others or at least compared to similar simulations it is necessary to provide sufficient and precise information for all of these points. Therefore, in the following we evaluate if this has been considered and what are the most commonly used options.

\subsection{Channel Models}

Figure 2 (left inset) depicts the distribution of channel model options used by the reviewed papers. Unfortunately in [13], there is no detailed discussion of this aspect, so we have no reference data available. Conspicuous
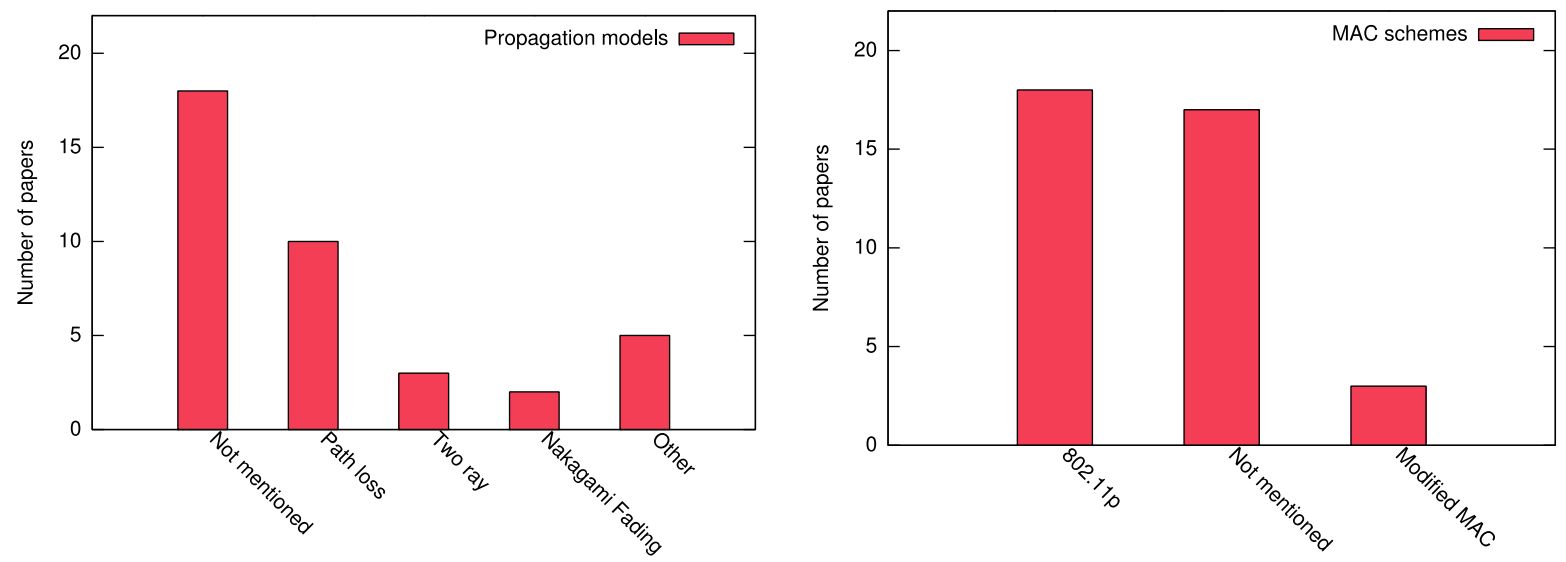

Figure 2. Left: The mentioned propagation models of the reviewed papers. This fact was not evaluated by [13], therefore, no reference data is available for previous years. Right: Used medium access schemes by the analyzed IVC simulations. Compared to [13] where lots of variants of IEEE 802.11 were used, the situation is much clearer now. Either the used MAC scheme was not mentioned or 802.11p was used and/or modified. Therefore, we did not include the reference data into this figure. 
is the fact that nearly half of all papers did not mention which model was used on the physical layer. This is precarious because this layer mainly influences the VANET characteristics and performance. In most of these cases, it can be assumed that a standard path loss model of the corresponding simulator for wireless communication networks was used. But most of these simulation frameworks offer several modules including parameters that could have been modified. Therefore, an accurate description with all parameters is still necessary, which is also true for all other alternative models to emulate the radio wave propagation.

The path loss model is mostly used, but it should be kept in mind that effects like fading, attenuation due to vehicles/buildings and multi path propagation, are not considered by this model. Depending on the environment, this model could still provide a sufficiently realistic emulation (e.g. for highways), but especially in case of an urban scenario where attenuation and multi path propagation could cause major effects, it would be worth to select a more detailed model of the radio channel. However, it must be pointed out that the pass loss model could be differently realized, e.g. attenuation could be a part of this model, but these aspects have not been mentioned by the publications in detail in most cases. Furthermore, some of them have even not defined the fundamental value of the path loss exponent. Therefore, considering that most publications have used a not proper described path loss model or even have not mentioned the channel model at all, it seems that these effects are handled as a less important part of IVS for most simulation studies.

The term "two ray" in Figure 2 (left inset) is a reference for a model discussed in [15]. It is an enhanced version of the path loss model especially considered for IVC. Nakagami propagation model was used by [16] [17]. Some channel models summarized under 'others' have used a more adequate approximation of real radio channel characteristics, preponderantly based on measurements in conjunction with MATLAB-models to evaluate medium access schemes or the basic performance attributes of VANET communications.

A detailed discussion of radio channel models for VANETs is out of the scope of this paper, therefore, we refer the interested reader to [18] and [19] for further details. The presented results and discussed attributes of the radio channel by these and other authors make clear, that a proper simulation of a VANET channel is difficult. However, to the best of our knowledge, we do not know any work that demonstrates the impact of a switch from a simple pass loss model to a more accurate channel emulation for the evaluation of VANET communication protocols especially on higher layers like routing or information dissemination schemes. Just [20] provides an evaluation with respect to common communication aspects. In general, these protocols are only indirectly influenced by special point to point communication characteristics, because they should work similar under different conditions, e.g. node density and network topology, but the performance will depend on the channel model, especially in urban scenarios. And even for highway simulations [21] measurements indicate a significant different behavior compared to simple path loss model. Therefore, we encourage further investigations on both aspects: IVC channel models and their influence on high layer IVC protocols; and not to utilize simple paths loss models.

\subsection{Medium Access}

The IEEE 802.11p standard has been approved in the year 2010. This explains why standards like IEEE 802.11a or IEEE $802.11 \mathrm{~b}$ were used in some publications reviewed by Joerer et al. Fortunately, the situation is much clearer in the year 2013. About every second paper indicates that the simulation is based on IEEE 802.11p. Not exactly edifying is the trend of not mentioning which medium access scheme was used. The average in the years 2009 to 2011 was about 20 percent whereas for the year 2013 it has increased to nearly 50 percent as shown by Figure 2 (right inset). The only explanation we have for this negative trend is that most network simulators already have a module for IEEE 802.11p. We would like to suggest authors to mention this option even in the case that this question should be indirectly clear. It should be considered that IEEE 802.11p defines several channels and it is advantageous not to simulate just one of these channels, as discussed by F. Klingler et al. [22].

Interesting is the fact that the work on modifications of the IEEE 802.11p medium access protocol keeps on a constant level. In most cases these publications evaluate a priority based access scheme by adjusting the contention window size for a more efficient forwarding scheme or for priority based beaconing. But some completely different approaches based on time division multiplex are discussed, too. Although the IEEE 802.11p standard has already been approved, the motivation and intention is understandable. The communication of a VANET is faced with serious problems in case of high node density, possible for urban or high way scenarios. The usual CSMA/CA algorithm has some drawbacks and the proposed alternatives could help that at least important vehicles (emergency vehicles) would be able to transmit messages. The adopted contention windows size seems to 
be an effective and simple solution for this problem. Examples for these works which were part of the papers we have evaluated are [23] and [24].

\subsection{Mobility Models and Scenarios}

Issues of channel models and medium access schemes are important for all kind of wireless networks, but additionally for VANETs, mobility models are a special additional challenge due to the need of an accurate realization of traffic flows. In this section we focus on the used software tools instead of specific vehicle following models like the Krauss Model or the Gipps Model. Furthermore, it should be kept in mind that there is a wide range of possible ways to implement such mobility models in VANET simulations, as presented in a comprehensive summary done by Härii et al. [25].

In general, a realistic modeling of vehicular mobility should consider effects on macroscopic and microscopic levels. An example for macroscopic effects is that the flow of traffic on a map is much higher on highways and main roads than on roads in residential areas, whereas microscopic effects take into account the individual behavior of a single vehicle, e.g. speed, acceleration or lane change decisions. The connection between macroscopic and microscopic issues is mainly given by the route each vehicle will take on a map. All software tools mentioned in Figure 3 (left inset) support microscopic effect, but the modeling of macroscopic effects is a problem, insufficiently realized at the moment. Additionally, looking at Figure 3 (right inset) we can see which scenario was simulated by the evaluated papers, which is closely related to this problem. For a highway scenario there is no need to consider classical macroscopic effects unless an unusual huge area with several highways is being simulated, which is not the case in any of these viewed publications.

The second most often used setup was an urban scenario modeled as a synthetic regular rectangular grid. Due to the similarities of such a grid to the road layout of downtown Manhattan (New York), it is commonly called Manhattan grid and indicated as 'Urban Manhattan' in Figure 3 (right inset). Although some roads of this grid could be defined as a main road and simulated with a higher vehicle density, this has not been done and a random generation of vehicles and routes has been used instead. Only 5 papers used a real map as a basis for the ITS evaluation. Thus, based on this literature survey, we have to say that macroscopic effects are not taken into account in most cases of ITS simulations. This is not necessarily a critical fact we should worry about. Some ITS communications parts like beaconing or medium access scheme are influenced by one-hop neighbor nodes of the VANET, therefore, for the evaluation of these protocols, it should be a valid simplification to ignore macroscopic effects. Just in case of multi-hop communication as in case of information dissemination schemes, these effects may have significant impact on the evaluation of these protocols. Unfortunately, to the best of our knowledge, there is no publication available which has investigated the effects and differences of choosing a Manhattan grid or areal word map with or without including macroscopic effects. Therefore, we would like to
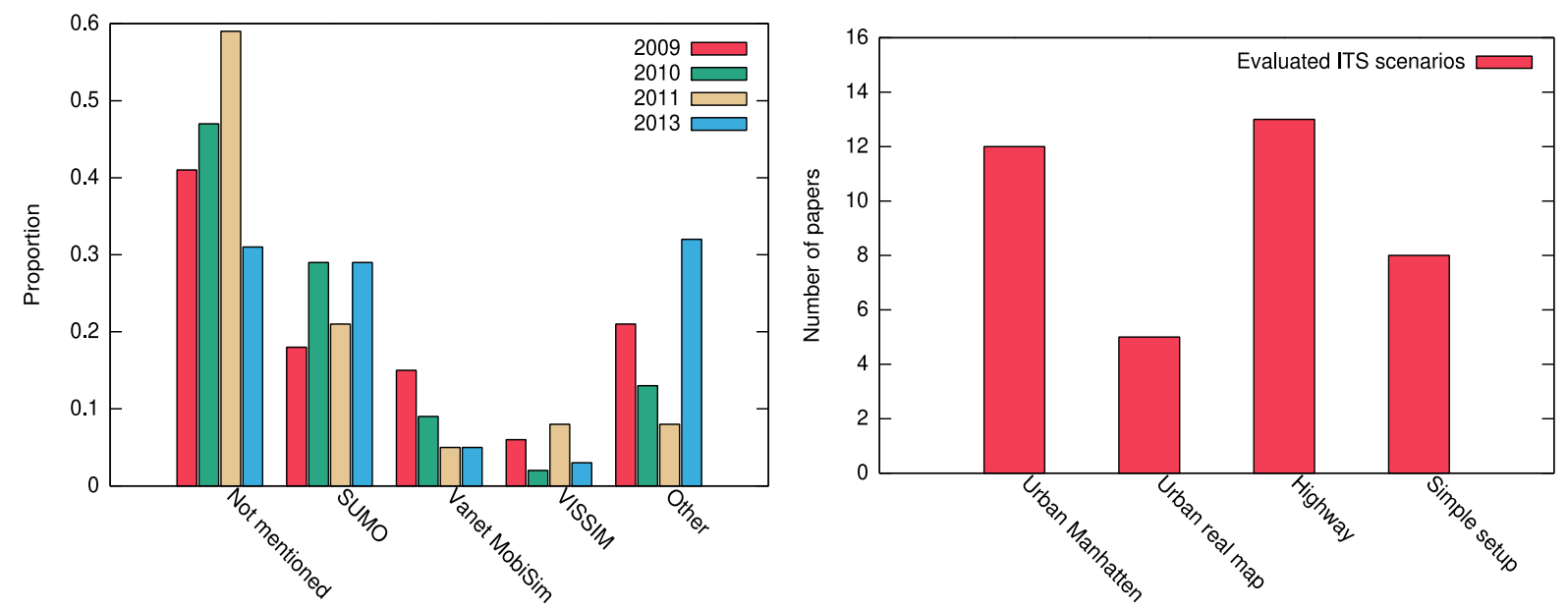

Figure 3. Left: Software tools which were used to emulate vehicle mobility. Summarized under "other": STRAW (two times), IDM CORSIM, VISSIM, MATSim (each once). Right: Simulated IVC scenarios, which are not evaluated by [13], therefore, we have no reference data for previous years. 
encourage researchers to take a closer look on macroscopic effects, how they can be modeled in different scenarios and to investigate if these effects have a significant impact on the evaluation of ITS communications protocols. Looking back to the left inset of Figure 3, we can see three major facts: 1) the amount of publications not indicating which mobility software tool or model was used for their IVC simulation has decreased. 2) SUMO is being used on a constantly high level. 3) Compared to SUMO the usage of alternative options is quite low.

Because SUMO seems to be the mostly used tool over several years, we want to mention some problems we were faced with during our work with SUMO. But we have to underline that in our point of view SUMO is a good software tool that really helps to perform ITS simulation, especially because it is free and open source software with an active community still enhancing its functionality.

- Based on the NETCONVERT tool, it is simple to import a real map from openstreetmap.org. But larger and more complex intersections are occasionally realized with more elements than needed. This problem especially occurs at roundabouts.

- It is possible to generate traffic light phases automatically. But this should only be seen as a basic setup, because on larger intersections or several traffic lights along a main road are in most cases not defined realistically. We also have to say that none of the reviewed papers had mentioned traffic lights. Therefore, we think these aspects were simply ignored in most cases unless adaptive traffic light schemes enabled by ITS communication were the key interest.

- It is difficult to simulate a scenario based on a real city map with a very high vehicle density. At an early stage of the simulation, vehicles will have to wait in front of some intersections due too many vehicles on the cross road, therefore, causing a growing traffic jam. In most situations this problem gets worse if the simulation continues. Certainly, this will happen in real situations as well, but we think this occurs at a too early stage in SUMO simulations. We have not looked at this problem in detail, but possible reasons are the lane changing process at a place where the number of lanes is decreased on a road or at road interchanges, as well as the merging process at intersections or highway entry points.

- The modeling of macroscopic effects still needs manual adjustments and random traffic flows generate rather an equal traffic density on the whole map than a significant higher traffic load on main roads.

But as said, we think SUMO is besides these problems a quite good traffic simulator and due to the active community it is likely to happen that these aspects get improved in the near future. Mobility traces [26] are an interesting alternative to evaluate IVC based on real traffic observations, one example is the TAPASCologne [27] project which was also used by one of the reviewed papers [28]. The drawback of such an approach is the lack of variability whereas pure random based movements do not emulate traffic flows realistically enough. The link between these options is given by mobility models based on activity definitions or a model emulating social behavior [29] [30]. Therefore, our point of view is that mobility traces are perfect for parameterization of those models but less suitable for direct usage in IVC simulations.

\subsection{Network Simulators}

Finally, the used network simulator is the central software element and influences which of the other software tools and models could be used for ITS simulations. The most common ones are the free and open source simulators NS-2, its successor NS-3 and OMNeT++, all on a comparable level, as depicted by Figure 4. The commercial tools OPNET and SWANS seem to be less important and the negative trend of the commercial tool QualNet has continued to a usage of zero in 2013. Summarized under others are further commercial simulation tools we do not mention separately.

In general, choosing one of these simulators has no direct influence on the results on simulations of IVC. All time triggered event simulators are suitable to emulate discrete systems like communication protocols. However, as we have discussed, ITS simulations consist of several elements and the choice of one of these simulators implies a large set of predefined modules and which interfaces to other applications like SUMO exist or could be implemented. For example, NS-3 offers more than 20 propagation models each with a set of predefined default parameters. Therefore, if the used network simulator was mentioned - which was not the case for almost one in four of the reviewed papers - and even the used propagation model was indicated, there would still be uncertainty left if the default parameters have been modified.

A detailed and comprehensive discussion of network simulators is given by [31], covering all sections from physical layer aspects up to mobility models and application issues. Most simulators are based on a modular 


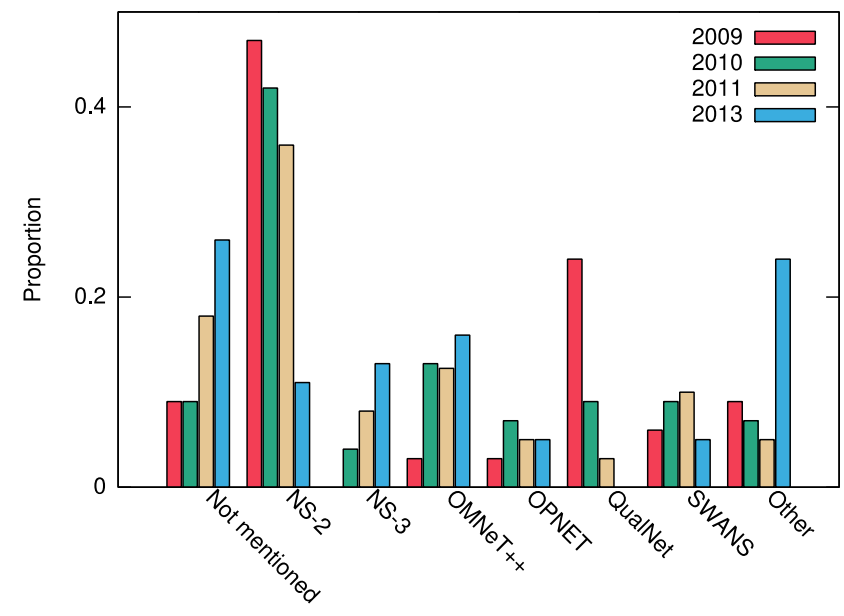

Figure 4. Distribution of network simulators used by the reviewed publications to perform IVC simulations.

structure allowing to extend their functionality with additional modules. In case of NS-2, NS-3 and Monnet++ the additional open source philosophy allows adapting and extending the software features to individual needs. Consequently, choosing one of these alternatives has no significant impact, but the including modules and their configuration should correctly be described to allow reproducing the presented simulation results.

\section{Conclusions}

Our study probably presents a less positive summary of ITS simulations publications compared to reality. We believe most researchers are certainly aware that the degrees of freedom we have discussed in Sections 3.1 to 3.4 are important to consider for IVC. Regarding the overall picture and besides some minor impreciseness, there are 30 percent or less of publications presenting their results on a too abstract level missing essential information. In most cases, the lack of information concerns propagation model or mobility model details.

Even though it is comprehensible that radio channel emulation is not in the main focus of IVC researchers, we think it is worth looking on this in more detail. Especially because there are studies presenting IVC channels in detail, whereas most simulations are performed based on simpler path loss models. It would be worth analyzing in more detail, if an accurate channel emulation is needed in case of lower level IVC protocols like medium access schemes or for higher level services like beaconing and information dissemination protocols or if and when it is a valid simplification to switch to a less complex propagation model.

Unfortunately, in case of mobility models, the degrees of freedom are much larger. First, the scenario to be evaluated must be chosen. If this scenario is an urban area, we can either select a Manhattan grid or a real map. Furthermore, either a random or more realistic definition of vehicle routes could be used and finally, the decision whether and how traffic lights should be included is the last option. Considering propagation models, there are two main questions: Which impact do realistic propagation models have on the various IVC protocols? In which case could a simple propagation model be used in order to reduce complexity? By considering traffic rules and different vehicles types (motor cycles, cars and trucks) or a reduced amount of IVC aware vehicles this model could be further refined, too.

For nearly all reviewed publications, it could be said that the simulated IVC protocol itself was clearly described and it should be no problem for other researchers to implement the proposed protocol logic. But due to the large set of parameters and scenario setup options of such simulations, it would be difficult to reproduce published results as an independent control. But our experience showed that researchers always acted very cooperatively if they were asked to share more details about published results.

In summary, based on the results of this survey, we would like to encourage more research to investigate effects of propagation models and different mobility model options on higher and lower level IVC protocols. We also would like to recommend mentioning the various details of IVC simulations more accurately in future publications and we hope that this work helps to consider the influencing factors of IVC carefully. 


\section{Acknowledgements}

The authors would like to thank the administration and members of "Thüringer Innovationszentrum Mobilität" (ThIMo)" and the group of researchers of "Powertrain-RadioTrain” (PORT) for their support and the Thüringer Aufbaubank TAB/ESF for their kind funding.

We also would like to thank Mr. Stefan Joerer for giving us his row data of his study for our comparison.

\section{References}

[1] Dar, K., Bakhouya, M., Gaber, J., Wack, M. and Lorenz, P. (2010) Wireless Communication Technologies for ITS Applications. IEEE Communications Magazine, 48, 156-162. http://dx.doi.org/10.1109/MCOM.2010.5458377

[2] Willke, T., Tientrakool, P. and Maxemchuk, N. (2009) A Survey of Inter-Vehicle Communication Protocols and Their Applications. IEEE Communications Surveys \& Tutorials, 11, 3-20. http://dx.doi.org/10.1109/SURV.2009.090202

[3] d’Orey, P. and Ferreira, M. (2014) ITS for Sustainable Mobility: A Survey on Applications and Impact Assessment Tools. IEEE Transactions on Intelligent Transportation Systems, 15, 477-493. http://dx.doi.org/10.1109/TITS.2013.2287257

[4] IEEE Standard (2010) Wireless LAN Medium Access Control (MAC) and Physical Layer (PHY) Specifications Amendment 6: Wireless Access in Vehicular Environments. IEEE Std. 802.11p.

[5] Kenney, J. (2011) Dedicated Short-Range Communications (DSRC) Standards in the United States. Proceedings of the IEEE, 99, 1162-1182. http://dx.doi.org/10.1109/JPROC.2011.2132790

[6] Kosch, T., Kulp, I., Bechler, M., Strassberger, M., Weyl, B. and Lasowski,R. (2009) Communication Architecture for Cooperative Systems in Europe. IEEE Communications Magazine, 47, 116-125. http://dx.doi.org/10.1109/MCOM.2009.4939287

[7] IEEE Vehicular Technology Society (2010) IEEE Standard for Wireless Access in Vehicular Environments (WAVE) Networking Services. IEEE Std. 1609.3.

[8] ETSI European Telecommunications Standards Institute (2011) ITS VC Basic Set of Applications Part 2: Specification of Cooperative Awareness Basic Service. ETSI TS 102 637-2.

[9] ETSI European Telecommunications Standards Institute (2010) ITS VC Basic Set of Applications Part 3: Specification of Decentralized Environmental Notification Basic Service. ETSI TS 102 637-3.

[10] Panichpapiboon, S. and Pattara-Atikom, W. (2012) A Review of Information Dissemination Protocols for Vehicular Ad Hoc Networks. IEEE Communications Surveys \& Tutorials, 14, 784-798.

[11] Stubing, H., Bechler, M., Heussner, D., May, T., Radusch, I., Rechner, H. and Vogel, P. (2010) SimTD: A Car-to-X System Architecture for Field Operational Tests. IEEE Communications Magazine, 48, 148-154. http://dx.doi.org/10.1109/MCOM.2010.5458376

[12] Festag, A., Le, L. and Goleva, M. (2011) Field Operational Tests for Cooperative Systems: A Tussle between Research, Standardization and Deployment. Proceedings of the 8th ACM International Workshop on Vehicular Inter-Networking, ser. VANET'11, New York, 19-23 September 2011, 73-78. http://dx.doi.org/10.1145/2030698.2030710

[13] Joerer, S., Sommer, C. and Dressler, F. (2012) Toward Reproducibility and Comparability of IVC Simulation Studies: A Literature Survey. IEEE Communications Magazine, 50, 82-88. http://dx.doi.org/10.1109/MCOM.2012.6316780

[14] Krajzewicz, D., Erdmann, J., Behrisch, M. and Bieker, L. (2012) Recent Development and Applications of SUMOSimulation of Urban Mobility. International Journal on Advances in Systems and Measurements, 5, 128-138. http://elib.dlr.de/80483/

[15] Sommer, C., Joerer, S. and Dressler, F. (2012) On the Applicability of Two-Ray Path Loss Models for Vehicular Network Simulation. 4th IEEE Vehicular Networking Conference (VNC 2012), Seoul, 14-16 November 2012, 64-69. http://dx.doi.org/10.1109/VNC.2012.6407446

[16] Javed, M. and Khan, J. (2013) A Cooperative Safety Zone Approach to Enhance the Performance of VANET Applications. IEEE 77th Vehicular Technology Conference (VTC Spring), Dresden, 2-5 June 2013, 1-5. http://dx.doi.org/10.1109/vtcspring.2013.6691819

[17] Al-Kubati, G., Al-Dubai, A., Mackenzie, L. and Pezaros, D. (2013) Fast and Reliable Hybrid Routing for Vehicular Ad Hoc Networks. 13th International Conference on ITS Telecommunications (ITST), Tampere, 5-7 November 2013, 20 25. http://dx.doi.org/10.1109/itst.2013.6685515

[18] Molisch, A., Tufvesson, F., Karedal, J. and Mecklenbrauker, C. (2009) A Survey on Vehicle-to-Vehicle Propagation Channels. IEEE Wireless Communications, 16, 12-22. http://dx.doi.org/10.1109/MWC.2009.5361174

[19] Sen, I. and Matolak, D. (2008) Vehicle-Vehicle Channel Models for the 5-GHz Band. IEEE Transactions on Intelligent Transportation Systems, 9, 235-245. http://dx.doi.org/10.1109/TITS.2008.922881 
[20] Gozalves, J., Sepulcre, M. and Bauza, R. (2012) Impact of the Radio Channel Modeling on the Performance of VANET Communication Protocols. Springer Telecommunications Systems, 50, 149-167.

[21] Schumacher, H. and Tchouankem, H. (2013) Highway Propagation Modeling in VANETs and Its Impact on Performance Evaluation. 10th Annual Conference on Wireless On-Demand Network Systems and Services, Banff, 18-20 March 2013, 178-185. http://dx.doi.org/10.1109/wons.2013.6578344

[22] Klingler, F., Dressler, F., Cao, J. and Sommer, C. (2013) Use Both Lanes: Multi-Channel Beaconing for Message Dissemination in Vehicular Networks. 10th Annual Conference on Wireless On-Demand Network Systems and Services (WONS), 18-20 March 2013, 162-169. http://dx.doi.org/10.1109/wons.2013.6578342

[23] Hsu, M.W. and Hsieh, T.Y. (2013) A Probability Based MAC Channel Congestion Control Mechanism for VANET. IEEE 77th Vehicular Technology Conference (VTC Spring), Dresden, 2-5 June 2013, 1-5. http://dx.doi.org/10.1109/vtcspring.2013.6691842

[24] Gaugel, T., Mittag, J., Hartenstein, H., Papanastasiou, S. and Strom, E. (2013) Indepth Analysis and Evaluation of SelfOrganizing TDMA. IEEE Vehicular Networking Conference (VNC), Boston, 16-18 December 2013, 79-86.

[25] Harri, J., Filali, F. and Bonnet, C. (2009) Mobility Models for Vehicular Ad Hoc Networks: A Survey and Taxonomy. IEEE Communications Surveys Tutorials, 11, 19-41.

[26] Pan, G., Qi, G., Zhang, W., Li, S., Wu, Z. and Yang, L. (2013) Trace Analysis and Mining for Smart Cities: Issues, Methods, and Applications. IEEE Communications Magazine, 51, 120-126. http://dx.doi.org/10.1109/MCOM.2013.6525604

[27] Uppoor, S., Trullols-Cruces, O., Fiore, M. and Barcelo-Ordinas, J. (2014) Generation and Analysis of a Large-Scale Urban Vehicular Mobility Dataset. IEEE Transactions on Mobile Computing, 13, 1061-1075. http://dx.doi.org/10.1109/TMC.2013.27

[28] Silva, F., Silva, T., Ruiz, L. and Loureiro, A. (2013) ConProVA: A Smart Context Provisioning Middleware for VANET Applications. IEEE 77th Vehicular Technology Conference (VTC Spring), Dresden, 2-5 June 2013, 1-5. http://dx.doi.org/10.1109/vtcspring.2013.6692488

[29] Petz, A., Enderle, J. and Julien, C. (2009) A Framework for Evaluating DTN Mobility Models. Proceedings of the 2nd International ICST Conference on Simulation Tools and Techniques, Rome, 2-6 March 2009, 94-101. http://dx.doi.org/10.4108/icst.simutools2009.5647

[30] Ekman, F., Keränen, A., Karvo, J. and Ott, J. (2008) Working Day Movement Model. Proceedings of the 1st ACM SIGMOBILE Workshop on Mobility Models, Mobility Models ’08, New York, 27-30 May 2008, 33-40. http://dx.doi.org/10.1145/1374688.1374695

[31] Wehrle, K., Güneş, M. and Gross, J. (Eds.) (2010) Modeling and Tools for Network Simulation. Springer, Heidelberg, Dordrecht, London and New York. 